\title{
DESIGN OF A VDF/TRFE COPOLYMER-ON-SILICON PYROELECTRIC SENSOR
}

\author{
D. Setiadi and P.P.L. Regtien*
}

Delft University of Technology, Department of Electrical Engineering

Mekelweg 4, $2628 \mathrm{CD}$ Delft, The Netherlands

*University of Twente, Department of Electrical Engineering

P.O. Box 217, 7500 AE Enschede, The Netherlands

\section{Abstract}

This paper presents a design of a VDF/TrFE copolymer-on-silicon pyroelectric sensor. For an optimal design of a VDF/TrFE-on-silicon pyroelectric sensor, the onedimensional diffusion equation is solved for the pyroelectric multilayer structure. The output current of the sensor is calculated. Improvement of the sensor can be obtained by : (a) etching the silicon substrate under the sensor element, and (b) an additional VDF/TrFE copolymer layer as thermal isolation. Different noise sources for a pyroelectric sensor have been calculated. The dielectric noise dominates the noise sources. This conclusion has been verified by measured values.

\section{Introduction}

Earlier, it has been suggested that the thinner the pyroelectric material, the better the voltage sensitivity of the detector [1]. A $1 \mu \mathrm{m}$ VDF/TrFE copolymer can be made by a spin coating technique. The pyroelectric properties of such a $1 \mu \mathrm{m}$ VDF/TrFE copolymer film are not essentially different from those of the thicker films [2]. Thin films offer the added advantage that the pyroelectric detector element can be directly integrated with IC technology. A new technology for a thinner, unstretched film of pyroelectric polymer is desired for high responsivity, uniform imaging and creating a pyroelectric smart sensor. The VDF/TrFE copolymer was reported to be pyroelectric without stretching [3]. This means that the VDF/TrFE copolymer is a suitable candidate for an imaging sensor.

Unfortunately, the substrate influences the voltage sensitivity of the pyroelectric detector. In literature, the effect of the substrate on the voltage sensitivity of the pyroelectric detector has been studied [4]. The numerical solutions demonstrated that the voltage sensitivity of a thin pyroelectric detector on a substrate is lower than that of a thicker detector.

The VDF/TrFE copolymer is deposited on a silicon substrate that will contain the readout electronics. The advantages of this pyroelectric smart sensor are ease of manufacturing and a large signal to noise ratio. A disadvantage is the decrease of sensitivity due to the silicon substrate. To diminish this disadvantage one must carefully design the pyroelectric sensor, in which the thicknesses of the VDF/TrFE copolymer, the silicon substrate, the thermal isolation and the electrodes must be considered. 


\section{Pyroelectric detector}

The VDF/TrFE copolymer pyroelectric sensor consists of 5 layers; two aluminium electrode layers, a pyroelectric (VDF/TrFE copolymer) film, and two substrate layers (silicon dioxide and p-type silicon). A thick masking dioxide layer reduces the parasitic thermal and electrical capacitance. The thickness of aluminium electrodes in a standard IC process is $600 \mathrm{~nm}$. Thermal conductivities, specific heats and densities of these materials are listed in Table 1.

The front face of the pyroelectric sensor has a heat loss per unit area of $g_{H} T_{1}(0)$ where $g_{H}$ is the radiation heat transfer coefficient and $T_{1}(0)$ is the temperature of the pyroelectric sensor relative to ambient. It is assumed that the heat loss per unit area of the back face of the pyroelectric sensor is $g_{N} T_{N}\left(w_{N}\right)$ where $T_{N}\left(w_{N}\right)$ is the temperature of the $\mathrm{N}$-th layer relative to ambient. It is also assumed that the heat capacity of an absorbing layer that is deposited on top of the pyroelectric sensor, is small in comparison with the heat capacity of the front electrode. $P$, is the incident radiation power per unit area, and is assumed to vary according to a sine wave; the absorption of the top layer is $\eta$.

\begin{tabular}{|l|c|c|c|}
\hline \multicolumn{1}{|c|}{ material } & $\begin{array}{c}\text { heat } \\
\text { conductivity }\left(\delta_{n}\right) \\
\text { W. } \mathrm{m}^{-1} \cdot \mathrm{K}^{-1}\end{array}$ & $\begin{array}{c}\text { specific heat } \\
\left(\mathrm{c}_{\mathrm{n}}\right) \\
\mathrm{J} \cdot \mathrm{kg}^{-1} \cdot \mathrm{K}^{-1}\end{array}$ & $\begin{array}{c}\text { density }\left(\mathrm{d}_{\mathrm{n}}\right) \\
\mathrm{kg} \cdot \mathrm{m}^{-3}\end{array}$ \\
\hline aluminium & 237 & 896 & 2707 \\
\hline VDF/TrFE copolymer & 0.1287 & 1223 & 1880 \\
\hline $\mathrm{SiO}_{2}$ & 1.3 & 750 & 2200 \\
\hline Silicon Substrate & 149 & 703 & 2330 \\
\hline
\end{tabular}

Table 1 The material parameters of the pyroelectric sensor

\section{Thermal diffusion equation}

The diffusion equation for each layer is

$$
\frac{\partial T(x, t)}{\partial t}=\frac{\delta_{n}}{c_{n} d_{n}} \frac{\partial^{2} T(x, t)}{\partial x^{2}}
$$

A solution of this differential equation is :

$$
T_{n}(x, t)=T_{n}(x) \exp (j \omega t)
$$

This yields

$$
T_{n}(x)=\frac{\delta_{n}}{j \omega c_{n} \sigma_{n}} \frac{\partial^{2} T_{n}(x)}{\partial x^{2}}
$$

The general solution of Eq (3) is 


$$
T_{m}(x)=A_{n} e^{\alpha_{n}\left(x-h_{n}\right)}+B_{n} e^{-\alpha_{n}\left(x-h_{n}\right)} \quad \text { with } \alpha_{n}=\sqrt{\frac{j \omega c_{n} d_{n}}{\delta_{n}}}
$$

Using the boundary conditions, the thermal diffusion equation for $\mathrm{N}$ layers, results in

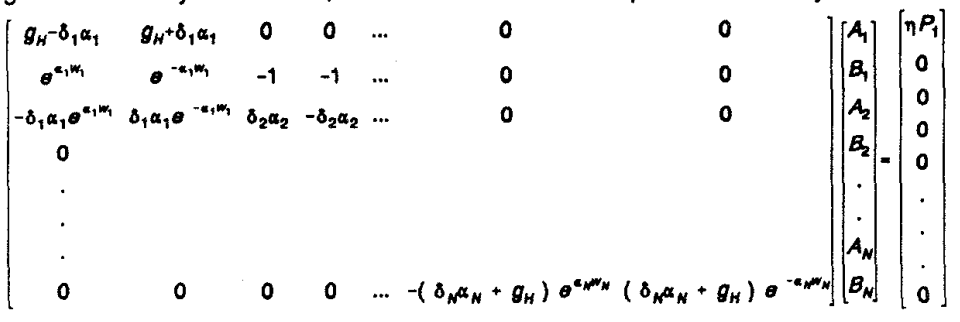

Now, one can use Eq (5) for the case of the pyroelectric sensor that consists of five layers. Using data of the material parameters of the pyroelectric sensor as shown in Table 1 , one can calculate the complex coefficients $A_{n}$ and $B_{n}$ as a function of frequency, by using gaussian elimination.

The spatial average of the temperature in the pyroelectric layer $\ddot{T}$ is:

$$
\bar{T}=\frac{\eta P_{1}}{\alpha_{2} w_{2}}\left(A_{2}\left(\theta^{\alpha_{2} w_{2}}-1\right)+B_{2}\left(1-\theta^{-\alpha_{2} w_{2}}\right)\right)
$$

where the coefficients $A_{2}$ and $B_{2}$ are a function of frequency, and can be calculated from Eq. (5).

\section{The output current}

If the pyroelectric coefficient is $p$ and the area of the pyroelectric sensor $A$, the temperature change will produce an alternating charge pAT, hence a current p A $\partial \overline{\mathrm{T}} / \mathrm{ot}$.

Using $\mathrm{Eq}(6)$, the output current is found to be

$$
\digamma_{0}=\frac{\eta P_{1} \rho A \omega}{\alpha_{2} w_{2}}\left(A_{2}\left(\theta^{\alpha_{2} w_{2}}-1\right)+B_{2}\left(1-e^{-\alpha_{2} w_{2}}\right)\right)
$$

In the next simulation, the incident radiation power per unit area $P_{1}$ is $1 \mathrm{~mW} / \mathrm{mm}^{2}$.

\subsection{The influence of the thickness of a VDF/TrFE copolymer film}

A model of a single VDF $T$ rFE copolymer pyroelectric sensor consists of $600 \mathrm{~nm}$ thick aluminium electrodes, a $1 \mu \mathrm{m}$ thick $\mathrm{SiO}_{2}$ and a $0.5 \mathrm{~mm}$ thick silicon substrate. The thickness of the VDF/TrFE copolymer is varied in the simulation: (a) $1 \mu \mathrm{m}$, (b) $10 \mu \mathrm{m}$, (c) $100 \mu \mathrm{m}$ and (d) $1 \mu \mathrm{m}$ without silicon substrate. Figure 1 shows the output current as a function of the frequency. It is found for the pyroelectric sensor with silicon substrate ( see lines (a), (b) and (c) of figure 1 ) that the current is constant 
below a certain frequency i.e. $20 \mathrm{~Hz}$ for the $1 \mu \mathrm{m}$ film. For a much thicker film, this frequency is lower. Below this frequency, the influence of the silicon substrate is noticeable. The current increases in proportion to frequency at much higher frequencies and it achieves a maximum at a frequency in which the thermal diffusion length equals the thickness of the film at that particular frequency, i.e, 34 $\mathrm{kHz}$ for the $1 \mu \mathrm{m}$ film. From the maximum frequency the heat does not fully penetrate into the VDF/TrFE copolymer. Therefore, in that region the current decreases with increasing frequency.
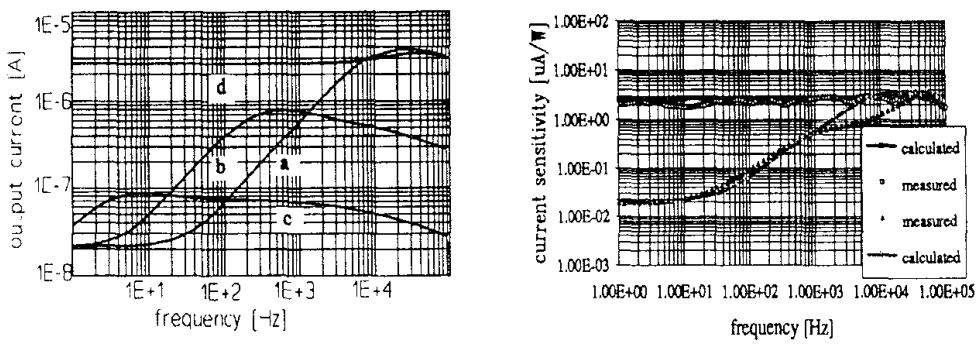

Figure 1 Effect of thickness of VDF/TrFE Figure 2 The measured and calculated copolymer on the output current current sensitivities as a function of frequency

\subsection{The silicon substrate}

Now, by etching the silicon substrate under the pyroelectric sensor element, one can neglect this substrate. From figure 1 line (a) and (d), it is found that the output current of the sensor is now much better than the sensor with the silicon substrate for the same thickness of the copolymer. So, a substantial improvement of a pyroelectric sensor can be obtained by etching the silicon substrate under the sensor element.

Figure 2 shows the measured and calculated current sensitivity as a function of the frequency. The first two lines ( $\square$ and *) show the measurement and the calculation values for the case of the sensor without the silicon substrate. The other two lines ( $\Delta$ and + ) show the measurement and the calculation values for the case of the sensor with the silicon substrate.

\subsection{The VDF/TrFE copolymer thermal Isolation}

An additional VDF/TrFE layer has been deposited between the back aluminium electrode and the silicon dioxide. This additional layer serves only as a thermal isolation. Therefore, it has not to be pyroelectric.

Figure 3 shows the effect of the thickness of the additional VDF/TrFE copolymer on the output current. A model of the pyroelectric sensor consists of $600 \mathrm{~nm}$ thick aluminium electrodes, a $1 \mu \mathrm{m}$ VDF/TrFE copolymer, a $1 \mu \mathrm{m}$ thick $\mathrm{SiO}_{2}$ and a 0.5 
$\mathrm{mm}$ thick silicon substrate. The thickness of the additional VDF/TrFE copolymer is varied in the simulation: (a) $1 \mu \mathrm{m}$, (b) $10 \mu \mathrm{m}$, (c) $100 \mu \mathrm{m}$. It is found that the frequency in which the output current achieves its maximum is still about $34 \mathrm{kHz}$ for any thickness of the additional copolymer. The additional copolymer improves the output current of the sensor, but the output current is still below that of the sensor without the silicon substrate (see line (d) of figure 3), especially in the low frequency range. The influence of the silicon substrate cannot be neglected.

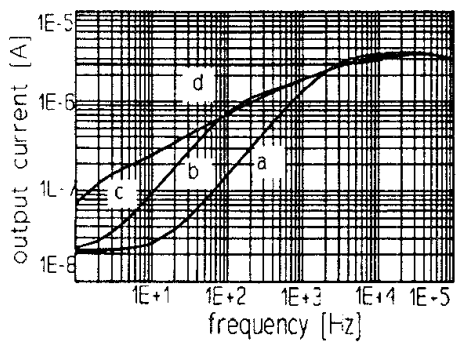

Figure 3 Effect of the additional VDF/TrFE copolymer on the output current

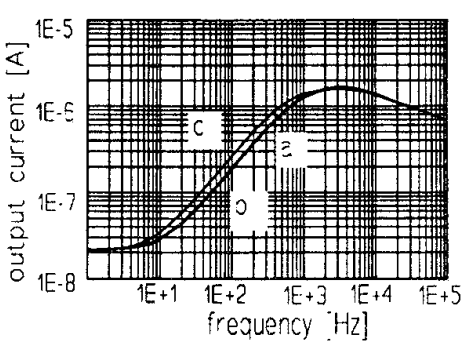

Figure 4 Effect of the silicon dioxide on the output current

\subsection{The effect of the silicon dioxide}

The silicon dioxide contributes not only to the total electrical capacitance of the pyroelectric sensor but also to the total thermal capacitance of the sensor. Figure 4 shows the effect of the silicon dioxide on the output current of the pyroelectric sensor for the cases: (a) a $0.1 \mu \mathrm{m} \mathrm{SiO} \mathrm{Sl}_{2}$ layer, (b) a $1 \mu \mathrm{m} \mathrm{SiO}{ }_{2}$ layer, (c) a $10 \mu \mathrm{m}$ $\mathrm{SiO}_{2}$ layer. The other parameters of the sensor are $600 \mathrm{~nm}$ electrodes, a $4 \mu \mathrm{m}$ $\mathrm{VDF} / \mathrm{TrFE}$ copolymer and a $0.5 \mathrm{~mm}$ silicon substrate. It has been found that the influence of the silicon dioxide on the output current of the pyroelectric sensor is small. The variation of the electrical capacitance, which is due to the thickness of a $\mathrm{SiO}_{2}$ layer, is a factor 100 from 0.1 to $10 \mu \mathrm{m} \mathrm{SiO}$ layer if the area of the pyroelectric sensor is constant.

\subsection{The influence of the aluminium electrodes}

The thickness of the electrodes, especially the front electrode, influences the output current of the pyroelectric sensor for high frequencies (above $1000 \mathrm{~Hz}$ ), as shown in Figure 5. Here, line (a) shows the pyroelectric sensor with a 600-nm aluminium front electrode and line (b) shows the pyroelectric sensor with a 100-nm aluminium front electrode. The other parameters of the pyroelectric sensor are a $4 \mu \mathrm{m}$ VDF/TrFE copolymer, a $1 \mu \mathrm{m}$ thick $\mathrm{SiO}_{2}$ and a $0.5 \mathrm{~mm}$ silicon substrate. The pyroelectric sensor with thin electrodes has the best response. Therefore, the electrode layers should be as thin as possible, especially the front electrode.

\section{Noise sources}


There are several noise sources in the pyroelectric sensor. The most important are: Johnson noise, thermal fluctuation noise and dielectric loss noise. From the calculation, it is found that the dielectric loss noise dominates the noise sources. This conclusion has been verified by the measurement values as shown in figure 6 . Figure 6 shows the current noise of a single pyroelectric sensor as a function of frequency (calculated value for the broken line and measurement values for the dashed line). It is found that the total current noise increases with increasing frequency, according to the nature of dielectric loss noise.

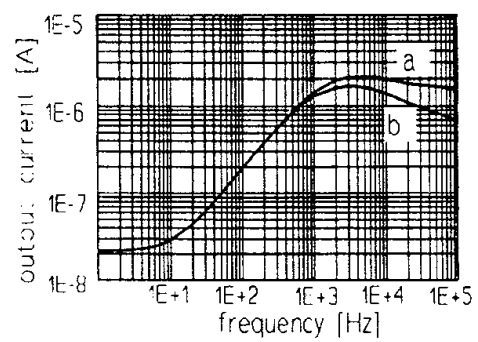

Figure 5 Effect of the top electrode on the output current

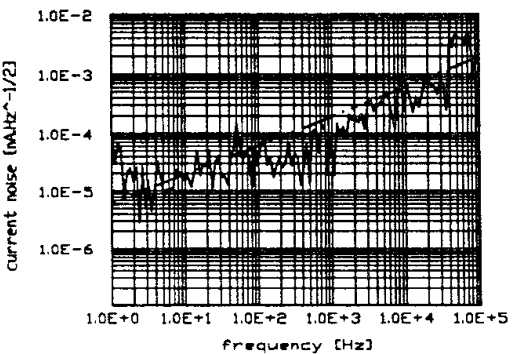

Figure 6 Measured and calculated current noise as a function of the trequency

\section{Conclusions}

The influence of the silicon substrate is measurable at low operation frequencies. The electrodes should be made as thin as possible, especially the front electrode. The silicon dioxide substrate hardly influences the output current of the pyroelectric sensor. Improvement of a pyroelectric sensor can be obtained by : (a) etching the silicon substrate under the sensor element, and (b) an additional VDF/TrFE copolymer as thermal isolation. The best improvement is obtained by etching the silicon substrate. The dielectric loss noise dominates the noise sources. The current noise increases with increasing frequency.

\section{Acknowledgements}

The authors would like to thank DIMES especially P.M. Sarro, Solvay \& Cie, Brussels, Belgium, T. $K w a$ and $M$. Wubbenhorst of Defft University of Technology.

\section{REFERENCES}

[1] E. H. Putley, Semiconductor and semimetal, Vol.5, ed. by Willardson and Beer, Academic Press, New York, 1970.

[2] K. Kimura, and H. Ohigasi, "Ferroelectric properties of poly (VDF/TrFE) copolymer thin film", Appt. Phys, Lett, Vol. 43, pp. 834-836, 1983.

[3] E. Yamaka, "Pyroelectric IR sensor using Vinylidene -Trifluoroethylene Copolymer Film", Ferroelectrics, Vol. 57, pp. 337-342, 1984.

[4] A. Van der Ziel, "Pyroelectric Response and D" of thin pyroelectric films on a substrate", J. Appl. Phys., Vol.44, No.2, pp. 546-549, 1973. 